\title{
SMRT Gate: A method for validation of synthetic constructs on Pacific Biosciences sequencing platforms
}

\author{
Rosalinda D'Amore ${ }^{1,2}$, James Johnson¹, Sam Haldenby², Neil Hall1,2,3, Margaret \\ Hughes $^{2}$, Ryan Joynson²,3, John G. Kenny², Nicola Patron³, Christiane Hertz-Fowler ${ }^{1,2}$, \\ and Anthony Hall ${ }^{1,2,3}$ \\ ${ }^{1}$ GeneMill, Centre for Genomic Research, MerseyBio Building, Institute of Integrative Biology, \\ University of Liverpool, Liverpool, UK, ${ }^{2}$ Centre for Genomic Research, Institute of Integrative \\ Biology, University of Liverpool, Liverpool, UK, and ${ }^{3}$ The Earlham Institute, Norwich Research \\ Park, Norfolk, UK
}

BioTechniques 63:13-20 (July 2017) doi 10.2144/000114565

Keywords: Golden Gate assembly; synthetic biology; next-generation sequencing (NGS); high-throughput; plasmid QC

Supplementary material for this article is available at www.BioTechniques.com/article/114565.

Current DNA assembly methods are prone to sequence errors, requiring rigorous quality control (QC) to identify incorrect assemblies or synthesized constructs. Such errors can lead to misinterpretation of phenotypes. Because of this intrinsic problem, routine QC analysis is generally performed on three or more clones using a combination of restriction endonuclease assays, colony PCR, and Sanger sequencing. However, as new automation methods emerge that enable high-throughput assembly, QC using these techniques has become a major bottleneck. Here, we describe a quick and affordable methodology for the QC of synthetic constructs. Our method involves a one-pot digestion-ligation DNA assembly reaction, based on the Golden Gate assembly methodology, that is coupled with Pacific Biosciences' Single Molecule, Real-Time (PacBio SMRT) sequencing technology.

Synthetic biology applies engineering principles to biology to build and optimize biological systems for new purposes using design-build-test cycles. Synthetic biology is a relatively new field with the potential to have a major impact on a large number of key areas, such as healthcare (1), agriculture, biofuels (2), novel materials (3), fine chemical production (4), bio-remediation (5), and clean water (6). A key component of synthetic biology is the ability to assemble and re-use biological parts. A powerful approach to assembly has been Golden Gate cloning $(7,8)$, which uses type IIS restriction enzymes and T4 ligase to assemble multiple parts in a single tube. A modular and hierarchical cloning system, MoClo (9), was developed that combines the concept of basic functional parts and Golden Gate technology, allowing the rapid assembly of complex constructs built up from basic parts. Fundamental to this strategy is that the parts and final constructs are sequenced to ensure correct ordering and high-fidelity synthesis.

Alongside this parts-based approach, another underpinning technology of synthetic biology is the application of high-throughput automation. Automated fabrication and testing allow reaction miniaturization, removing human error and increasing experiment speed and scale (10). However, the scale of production of DNA assembly now creates another bottleneck: quality control (QC) of the final products. Routinely, Sanger sequencing is used to perform $\mathrm{QC}$ of the assembled constructs. Three or more positive clones are screened using a combination of colony PCR and restriction endonuclease assays to verify the correct size of the assembled constructs, and these clones are subsequently sequenced to confirm that the parts were assembled correctly. Sanger sequencing involves designing and ordering primers that allow sequencing to span the full length of the construct and submitting

\section{METHOD SUMMARY}

Here, we present a protocol for screening of multipart synthetic constructs using the Golden Gate assembly method to build barcoded libraries combined with the long-read sequencing capability of Pacific Bioscience RSII sequencing platform. 
Table 1. Experimental design: $5^{\prime}$ and $3^{\prime}$ overhangs generated after digestion with $B b s l$ enzyme.

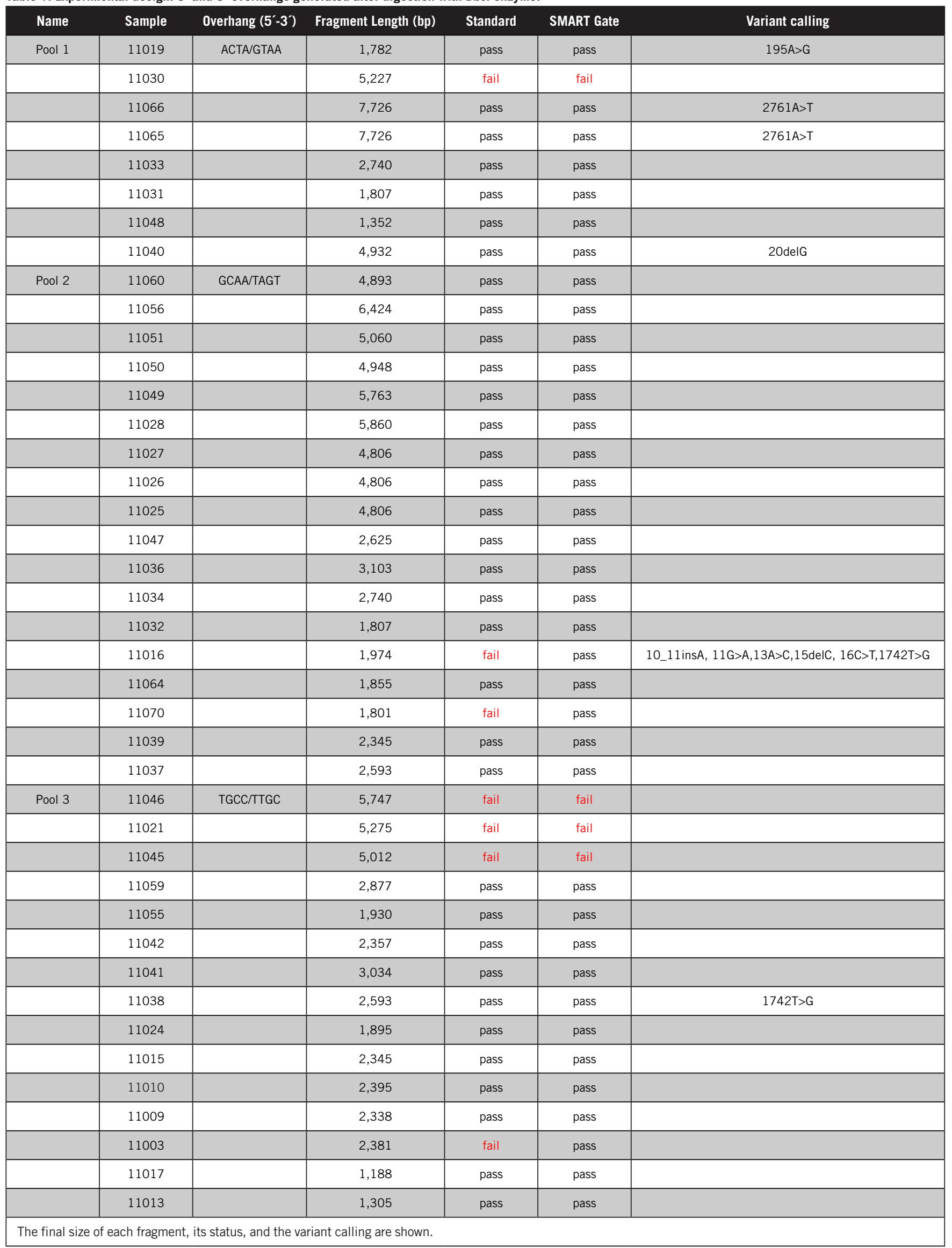


each construct to multiple Sanger sequencing reactions to achieve a full $2 \times$ coverage sequence. This is an expensive, time-consuming and difficult to automate approach.

Here, we describe a new methodology (SMRT Gate) that takes advantage of the long-read technology offered by the Pacific Biosciences (PacBio) RSIl sequencing platform (Pacific Biosciences, Menlo Park, CA) (11) in combination with a type IIS restriction enzyme-based cloning system to rapidly attach sequencing barcodes and adapters to generate a barcoded library suitable for PacBio sequencing.

PacBio long-read sequencing technology captures sequence information during replication of the target DNA molecule. The template, called a SMRTbell, is a closed, single-stranded circular DNA that is created by ligating hairpin adaptors to both ends of the target double-stranded (dsDNA) linear DNA (12).

PacBio protocols exist to build SMRTbell libraries with insert sizes ranging from $500 \mathrm{bp}$ to $>20 \mathrm{~kb}$. Although barcoding methods do exist, these are time-consuming and involve multiple steps.

SMRT Gate is based on Golden Gate MoClo (9). The Golden Gate Cloning method allows basic genetic elements to be made into standardized DNA parts by cloning them into a plasmid vector (Level 0). Subsequently, these standard parts are assembled together in a single step to build transcriptional units (in Level 1 plasmid vectors), which have defined fusion sites and positions for further assembly. Level 1 transcriptional units can be assembled

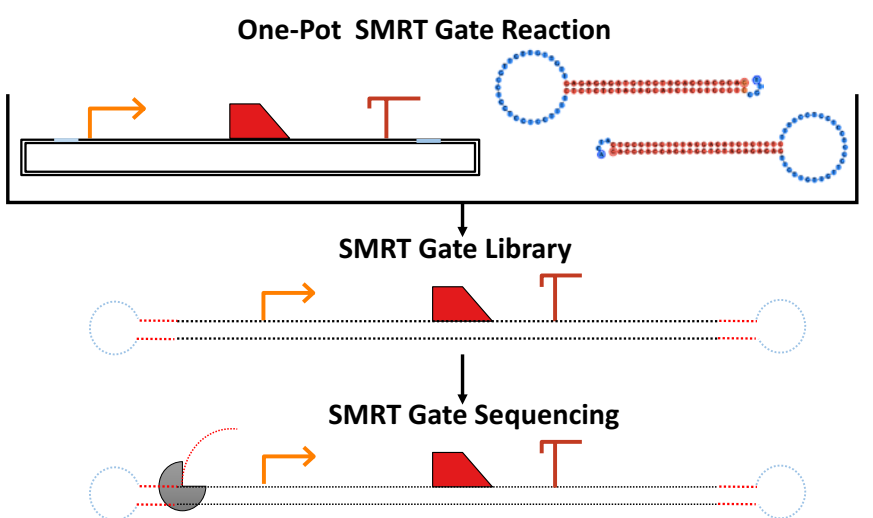

Figure 1. Schematic of SMRT Gate library preparation and sequencing reaction. ed DNA (dsDNA) fragments are generated using $B b s$ (Bpil) restriction sites (light blue) and are thus released from the backbone. The barcoded (red) SMRT Gate hairpin adapters (blue) are ligated onto the dsDNA fragments using the overhang generated by the cleavage. The DNA polymerase (gray) was bound to the SMRT library to generate polymerase reads (red). Dashed lines represent single-stranded DNA (ssDNA). The orange arrow (promoter), red trapezoid (coding region), and brown $\mathrm{T}$ (terminator) represent the components of the gene construct.

up to six at a time in the next assembly levels (Level 2 or M). All MoClo assemblies are free of internal recognition sequences for Bbsl (Bpil) and Bsal, which flank the parts and are used for iterative assembly.

\section{The SageHLS Hmw Library system \\ - Extract HMW DNA from cell suspensions \\ - Collect fragments up to $2 \mathrm{MB}$ \\ - HLS-CATCH ${ }^{\mathrm{m}}$ - isolate genomic regions with CRISPR/Cas9 \\ - Less than 1 day sageHLS workflow, minutes of hands-on time}

for Long-Range Genomics

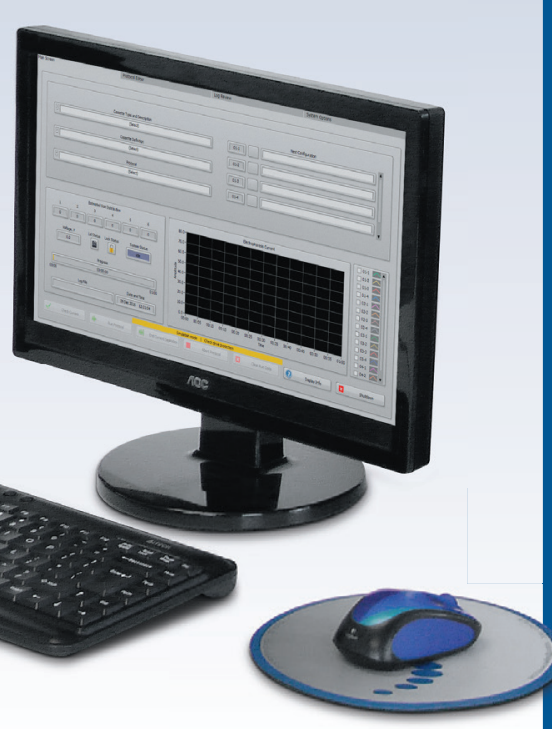



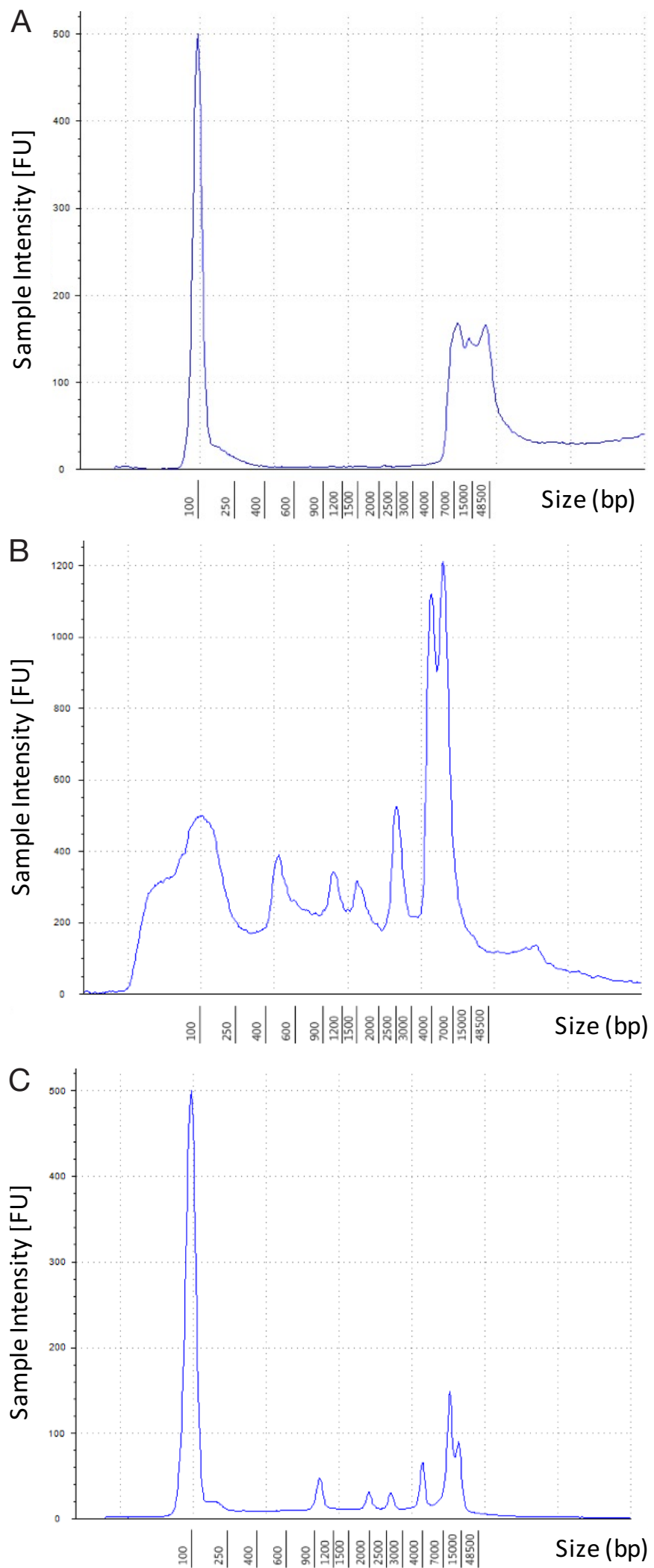

Figure 2. QC Tapestation electropherograms of the SMRT Gate library (Pool 1). Electropherogram before SMRT Gate reaction (A), before exonuclease treatment (B), and of the final SMRT Gate library (C) are shown. The SMRT Gate library showed 6 distinct peaks (1300, $1800,2700,4500,7700$, and $9700 \mathrm{bp}$ ) comparable with the size of the fragments released from the constructs in Pool 1 after cleavage.
Our methodology becomes very efficient for highthroughput construct screening, and it is cost-effective compared with Sanger sequencing when a batch of 12 constructs ( $>5 \mathrm{~kb}$ each) is multiplexed in a single PacBio run (Supplementary Table S1).

We used the SMRT Gate methodology to sequence 41 synthetic constructs (insert size ranging 1.3-7.7 kb) built using the MoClo Assembly Standard (9).

\section{Materials and methods}

SMRTbell adapter

The oligonucleotides (5' phosporylated and PAGE purified) that are used to generate the SMRTbell adapter were ordered from IDT (Leuven, Belgium) (Supplementary Table S2). The SMRTbell adapter must be annealed to form a hairpin prior to ligation. To anneal the SMRTbell adapter, the oligonucleotides were resuspended in $1 \times$ annealing buffer (10x annealing buffer: $100 \mathrm{mM}$ Tris- $\mathrm{HCl} \mathrm{pH} 7.5,5 \mathrm{M} \mathrm{NaCl}$ ) at a final concentration of $20 \mu \mathrm{M}$ in $20 \mu \mathrm{l}$. The annealing reactions were incubated at $80^{\circ} \mathrm{C}$ for $2 \mathrm{~min}$, then ramped down to $25^{\circ} \mathrm{C}$ at a rate of $0.1^{\circ} \mathrm{C} / \mathrm{s}$ before transferring to $4^{\circ} \mathrm{C}$ for immediate use or storage at $-20^{\circ} \mathrm{C}$.

Generation of SMRT gate library

Forty-one plasmids (see Table 1 for a summary of the experimental design) were assembled in three pools using an equimolar amount of each plasmid. Each pool (500-1000 ng) was subjected to a Golden Gate-type reaction to digest and ligate the barcoded SMRT Gate SMRTbell as follows: $1 \mu$ l T4 ligase (1 U/ul, ThermoFisher Scientific, Runcorn, UK), $1 \mu \mathrm{l} 10 \mathrm{nM}$ ATP [New England Biolabs (NEB), Hitchin, UK], $0.5 \mu \mathrm{l} \mathrm{Bbsl}(10,000 \mathrm{U} / \mathrm{ul})$ (NEB), $5 \mu \mathrm{l}$ 10x Buffer 2.1 (NEB), $1 \mu$ I SMRT Gatebell (Supplementary Table S2) a and b $(20 \mu \mathrm{M})(\mathrm{IDT})$ in a final reaction volume of $50 \mu \mathrm{l}$ or scaled down to $10 \mu \mathrm{L}$ when automation was used. The reaction was incubated on a thermocycler (Agilent SureCycler 8800; Agilent Stockport, UK) as follows: first step at $37^{\circ} \mathrm{C}$ for 20 $\mathrm{min}$, second step at $37^{\circ} \mathrm{C}$ for $1.5 \mathrm{~min}$ and $16^{\circ} \mathrm{C}$ for $5 \mathrm{~min}$ (15 cycles) and then $50^{\circ} \mathrm{C}$ for 5 min and finally incubation at $80^{\circ}$ for $10 \mathrm{~min}$. After ligation, the reaction was exonu-

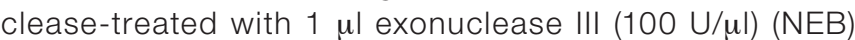

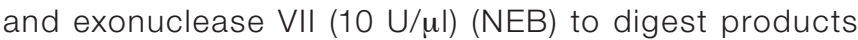
that failed ligation as well as templates containing internal nicks. The libraries were cleaned up using $1 \times$ AMPureXP Beads (Beckman, High Wycombe, UK) and resuspended in $10 \mu \mathrm{l} \mathrm{TE}$ buffer. One microliter of each library was run on Agilent Genomic DNA ScreenTape to ensure that the final product was the correct size and then quantified using a Qubit (ThermoFisher Scientific) dsDNA HS assay. The 3 libraries were pooled, and the concentration of the final library was $5 \mathrm{ng} / \mathrm{\mu l}$.

\section{Standard library}

The same pools of constructs were prepared as described above. Each pool was restriction digested using $1 \mu \mathrm{l}$ Bbsl $(10,000 \mathrm{U} / \mathrm{ul}), 5 \mu \mathrm{l} 10 \times$ Buffer 2.1 at $37^{\circ} \mathrm{C}$ for $60 \mathrm{~min}$ in a final volume of $20 \mu \mathrm{l}$. Two microliters of rSAP (NEB) was then added to the reaction, which was incubated at $37^{\circ} \mathrm{C}$ 
for $60 \mathrm{~min}$ and subsequently at $65^{\circ} \mathrm{C}$ for $5 \mathrm{~min}$. Ligation was performed by adding $1 \mu \mathrm{l}$ T4 ligase $(1 \mathrm{U} / \mu \mathrm{l}), 1 \mu \mathrm{l} 10 \mathrm{nM}$ ATP, $5 \mu \mathrm{l} 10 \times$ Buffer 2.1, $1 \mu \mathrm{l}$ barcoded SMRTbell a and b (20 $\mu \mathrm{M}$, followed by incubation at $25^{\circ} \mathrm{C}$ for $60 \mathrm{~min}$. A clean-up step was performed using 1× AMPureXP Beads (Beckman) and eluted in $10 \mu \mathrm{l}$ of TE buffer. The repair reaction was performed using PreCR Repair Mix (NEB) according to the manufacturer's instructions. After the repair step, the library was exonuclease treated and cleaned up as described above. The final library concentration was $8 \mathrm{ng} / \mu \mathrm{l}$.

\section{PCR verification}

Constructs 11016, 11030, 11046, 11027, and 11045 (5 ng each) were used to PCR amplify the inserts with 0.3 $\mu \mathrm{M}$ Level1TDNAL_for (IDT), $0.3 \mu \mathrm{M}$ Level1 RB_rev (IDT) (Supplementary Table S2), and 1× OneTaq Hot Start DNA polymerase ready mix (NEB). The PCR for each variable region was carried out in triplicate in a $25 \mu \mathrm{l}$ reaction in the thermal cycler with the following parameters: initial denaturation at $94^{\circ} \mathrm{C}$ for $5 \mathrm{~min}$, followed by 25 cycles of $98^{\circ} \mathrm{C}$ for 20 $\mathrm{s}, 60^{\circ} \mathrm{C}$ for $15 \mathrm{~s}$, and $72^{\circ} \mathrm{C}$ for $3 \mathrm{~min}$, with a final extension at $72^{\circ} \mathrm{C}$ for $3 \mathrm{~min}$.

\section{Sanger sequencing}

Five microliters purified plasmid DNA (80 - $100 \mathrm{ng} / \mathrm{ul}$ ) was mixed with $5 \mu \mathrm{L}$ primer leverl1TDNAL_for or Level1 RB_rev (5 $\mu \mathrm{M})$ and submitted to sequencing provider GATC (Konstanz, Germany) for Sanger sequencing.

\section{PacBio Sequencing}

The Pacific Biosciences Binding Calculator was used to determine the amount of primers and polymerase needed for the binding reactions, based on an insert size of 4500 bp. The primers and Pacific Biosciences proprietary p6 SA DNA polymerase $v 2$ were bound to the library and then the MagBead Kit (Pacific Biosciences) was used to bind the library complex with MagBeads before sequencing to reduce adapter dimers. The MagBeads/SMRTbell-polymerase complexes were loaded into a 96-well plate. The plate, along with a DNA Sequencing Kit 6.0 (Pacific Biosciences) was loaded onto the instrument. Each SMRT cell was loaded with a single binding complex, and 360 min movies were collected on a Pacific Biosciences RSII sequencer.

\section{Sequencing data analysis}

The sequencing data generated by the Pacific Biosciences RSII sequencer was analyzed using the RS_resequencing pipeline in the onboard software provided in the SMRT portal (software v2.2; https://github.com/PacificBiosciences/ SMRT-Analysis/wiki/SMRT-Analysis-Software-Installationv2.2.0) using the construct design sequences as a reference file. The depth of coverage across the constructs was determined and plotted using the BED format file produced by the PacBio portal, facilitating identification of regions of construct coverage dropout. SNPs and indels were called as part of the SMRT portal RS_Resequencing workflow after consensus sequence identification using Quiver (https:// github.com/PacificBiosciences/GenomicConsensus).
Q11019 T T T A T A T G T G A A A T A R11019 T T T A T A T A T G A A A T A S11019 T T T A T A T G T G A A A T A

Q11065 A T T A T A T T G A G G A G T R11065 A T T A T A T A G A G G A G T

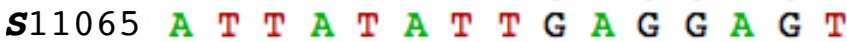

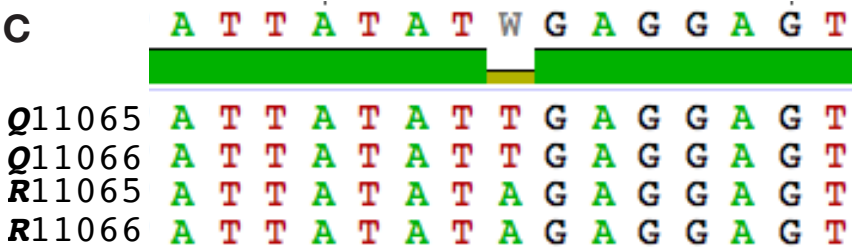

Figure 3. Example of variant calling. Alignment of 11019 Quiver (Q11019) and Sanger (S11019) reads mapped to the reference sequence (R11019) showed a SNP at position 208 (A). Alignment of Quiver consensus (Q11065, Q11066) and reference sequences (R11065, R11066) for 11065 and 11066 showed a new variant (B) and concordance in SNP calling (C).

\section{Results and discussion}

SMRT Gate library construction

For the SMRT Gate method, we designed the SMRTbell adapters (Figure 1) using the unique fusion sites generated by cleavage of Level 1 constructs with Bbsl (Bpil). Forty-one Level 1 synthetic constructs were pooled into three groups based on their fusion sites (Table 1). The three pools were processed individually, using either the barcoded SMRT Gatebells (Supplementary Table S2), Bbsl (Bpil) and T4 DNA ligase or the SMRTbells with the standard multi-step library protocol, to generate three barcoded SMRTbell libraries for PacBio sequencing for each method.

Libraries were analyzed using a TapeStation to assess fragment size and yield. The fragment profiles for both methods were comparable, but the SMRT Gate libraries had a lower yield (50 ng) compared with the standard library (80 ng). Prior to going through the SMRT Gate protocol, libraries showed a single peak (Figure 2A; see also panel 1 of Supplementary Figures S1 and S2) comparable to the size of the initial synthetic constructs pool; however, after cleavage with BbsI and SMRTbell ligation, libraries showed distinct peaks comparable in size to the fragments released from constructs in each pool after cleavage, and they also showed a smear between $50 \mathrm{bp}$ and $45 \mathrm{~kb}$ that represented unligated SMRTbells and undigested constructs (Figure 2B; see also panel 2 of Supplementary Figures S1 and S2). To reduce the amount of unligated SMRTbells and undigested synthetic constructs, an exonuclease reaction was performed on both libraries. After performing the exonuclease reaction, the final SMRT Gate library had a reduced background. The peaks correspond to the expected fragment sizes generated after endonuclease cleavage for 
each pool (Figure 2C; see also panel 3 of Supplementary Figures S1 and S2).

The better yield of the standard protocol did not translate into increased sequencing throughput. Failure of the SMRTbell ligation and exonuclease reactions were the likely cause of the loading bias. The low sequencing yield was due to under-loading, where the majority of wells of the SMRT cell did not contain a template.

\section{Analyzing data using the SMRT portal}

Raw reads generated by the RSII PacBio sequencing platform were analyzed using the on-board tools at the SMRT portal, a cloud-based software platform (www.pacb.com/support/ software-downloads/) The data sets were analyzed using the RS_Resequencing pipeline. Briefly, length- and quality-filtered reads were de-multiplexed using the Resequencing Barcoding module and subsequently locally aligned to the reference sequence based on the construct designs using BLASR. Following mapping, the Quiver algorithm was employed to detect variants and generate consensus sequences. An overview of variant calling and coverage for all of the constructs for both methodologies is provided in Table 1 and Supplementary Table S3. We sequenced 41 synthetic constructs, with an average depth of coverage of 13,162-fold for the SMRT Gate constructs and 878-fold for the standard library. The depth of sequencing for each construct was unbalanced, ranging from 12- to 57,120-fold for the SMRT Gate constructs and 2- to 4565-fold for the standard protocol. Although the constructs were prepared with consideration given to fragment sizes in the pooling calculation, consistent with our previous experience with the Pacific Biosciences RSII, small fragments were still preferentially loaded over larger ones. Loading of SMRTbell template is size-dependent, which is particularly important when sequencing libraries have different insert sizes. To overcome this issue and to achieve a more even representation we highly recommend selecting libraries of a similar size $( \pm 10 \%)$ to minimize loading biases or increasing the concentration of larger fragments in the pools (www.pacb.com/wp-content/uploads/2015/09/Guide-PacificBiosciences-Template-Preparation-and-Sequencing.pdf).

Analysis pipeline and variant calling

Considering that the average for polymerase reads in PacBio sequencing is $50 \mathrm{~kb}$ and that the maximum fragment length in the pool is $7.7 \mathrm{~kb}$, we expected the reads to map across the entire length of the synthetic construct.

After mapping and variant calling, we filtered out the four constructs (11021, 11030, 11045, 11046; indicated as FAIL in Table 1) that had a drop in local coverage across the reference sequence (within a 200-bp window) in spite of their overall coverage being $>20$-fold. For these constructs, we used a combination of PCR and Sanger sequencing. For the PCR, we designed primers to target the vector sequence and amplify across the inserts, and we Sanger-sequenced the amplicon to verify its sequence. We confirmed that the constructs provided were different from the reference sequence and, therefore, looked as if they had failed because we had not included a reference sequence for these constructs in our reference mapping database.
Of the remaining 37 constructs, the PacBio sequence data agreed $100 \%$ with the reference sequence for 31 constructs. In six constructs, the RS_resequencing pipeline identified five SNPS and four indels in the constructs generated via SMRT Gate protocol, while four SNPs and three indels were identified in the constructs generated via the standard method (Table 1 and Supplementary Table S3). All the variants were confirmed by Sanger sequencing and demonstrated that they must have occurred during the assembly of the original Level $O$ parts.

Part of the screening process is selecting two to five colonies and sequencing them to assess point mutations. To confirm the ability of the PacBio platform to discriminate single point mutations, we included two constructs in the pool with a single base change at position 3332 (11065 T/11066 C). The reference sequences for 11065 and 11066 were aligned to the consensus sequences generated by Quiver (Figure 3A, left panel) and the software correctly assigned the SNP to position 3332. Moreover, a new SNP in position 2774 was found (Figure 3A, right panel) and subsequently validated.

SMRT Gate can be used to generate libraries suitable for single-molecule sequencing technology without the need for individual barcoding and time-consuming procedures. Although we have yet to test the methodology on constructs made using other type IIS assembly methods, it could also be applied to those made using BASIC (13), Golden Braid (14), or TNT (15). The methodology can be adapted to any construct flanked by recognition sequences for type IIS restriction endonucleases that generate a 4-bp overhang. The RSII PacBio platform can generate up to 100,000 reads, while the Sequel can produce 5 times more reads. In our experiment, the uneven distribution was probably due to loading bias, which can be mitigated by sequencing libraries within the same size range $( \pm 10 \%)$.

If library size is considered, the read distribution should be more even; theoretically a coverage of up to $260 \times$ can be achieved when multiplexing 384 samples per SMRT cell on a RSII PacBio platform (based on 100,000 reads per run). That would provide enough coverage for calling variants with confidence and be a less expensive alternative to Sanger sequencing.

\section{Author contributions}

L.D. and A.H. conceptualized the study. L.D. performed the experiments and interpretation of the data. J.K. and J.J.reviewed the experiment design. L.D. and M.H. performed the sequencing experiments. S.H. and R.J. assisted with bioinformatic analyses. N.P., A.H., C.H.F., and N.H. assisted in the data interpretation. L.D. and A.H. wrote the manuscript. All authors read and approved the paper.

\section{Acknowledgments}

Sequencing was performed at the Centre for Genomic Research at the University of Liverpool. All plasmids were provided by N.P. L.D. and A.H. were funded by a BBSRC grant (BB/M00094X/1), and the Pacific Bioscience RS II 


\section{STATE OF THE ART SYSTEM FOR GEL AND BLOT IMAGING}

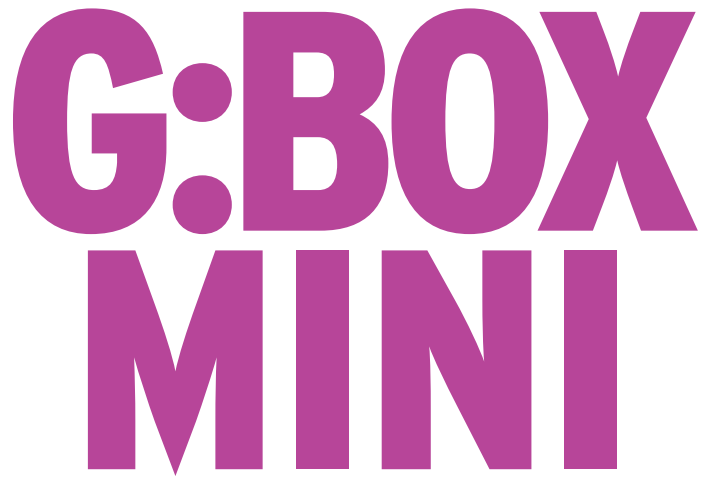

FOR ALL YOUR APPLICATION NEEDS

Suitable for chemiluminescence, fluorescence, DNA, multiplexing western blots or gels

Faster imaging with HI-LEDs to improve workflow Takes up less lab space

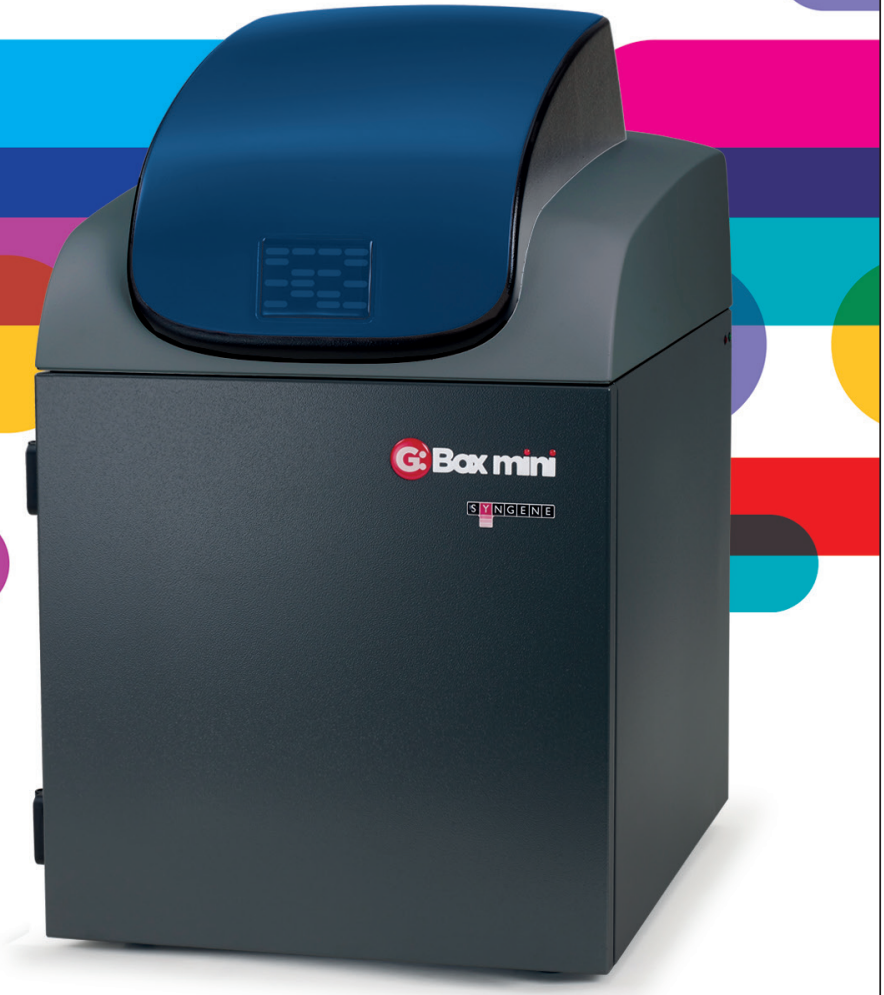

For further information, please contact ussales@syngene.com www.syngene.com/gbox-mini-gel-doc

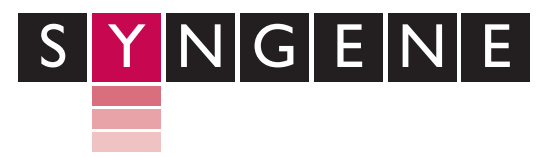

was purchased with a capital grant from the BBSRC (BB/ L014777/1). We would like to thank Chris Lounds and Adam Peltan from New England Biolabs for advice and recommendation on enzymatic assays, John Harting from Pacific Biosciences for the advice on bioinformatics tools, and Hannah McCue fromm the GeneMill team for her support.

\section{Competing interests}

The authors declare no competing interests.

\section{References}

1. Slomovic, S., K. Pardee, and J.J. Collins. 2015. Synthetic biology devices for in vitro and in vivo diagnostics. Proc. Natl. Acad. Sci. USA 112:14429-14435.

2. Georgianna, D.R. and S.P. Mayfield. 2012. Exploiting diversity and synthetic biology for the production of algal biofuels. Nature 488:329335.

3. Bryksin, A.V., A.C. Brown, M.M. Baksh, M.G. Finn, and T.H. Barker. 2014. Learning from nature - Novel synthetic biology approaches for biomaterial design. Acta Biomater. 10:1761-1769.

4. Keasling, J.D. 2012. Synthetic biology and the development of tools for metabolic engineering. Metab. Eng. 14:189-195.

5. Zhang, W. and D. Nielsen. 2014. Synthetic biology applications in industrial microbiology. Front. Microbiol. 5:451.

6. Molyneux-Hodgson, S. and A.S. Balmer. 2014. Synthetic biology, water industry and the performance of an innovation barrier. Sci. Public Policy 41:507-519.

7. Engler, C., R. Kandzia, and S. Marillonnet. 2008. A One Pot, One Step, Precision Cloning Method with High Throughput Capability. PLoS One 3:e3647.

8. Engler, C., R. Gruetzner, R. Kandzia, and S. Marillonnet. 2009. Golden Gate Shuffling: A One-Pot DNA Shuffling Method Based on Type Ils Restriction Enzymes. PLoS One 4:e5553.

9. Weber, E., C. Engler, R. Gruetzner, S. Werner, and S. Marillonnet. 2011. A Modular Cloning System for Standardized Assembly of Multigene Constructs. PLoS One 6:e16765.

10. Kanigowska, P., Y. Shen, Y. Zheng, S. Rosser, and Y. Cai. 2016. Smart DNA Fabrication Using Sound Waves: Applying Acoustic Dispensing Technologies to Synthetic Biology. J. Lab. Autom. 21:49-56

11. Eid, J., A. Fehr, J. Gray, K. Luong, J. Lyle, G. Otto, P. Peluso, D. Rank, et al. 2009. Real-Time DNA Sequencing from Single Polymerase Molecules. Science 323:133-138.

12. Roberts, R.J., M.O. Carneiro, and M.C. Schatz. 2013. The advantages of SMRT sequencing. Genome Biol. 14:405.

13. Storch, M., A. Casini, B. Mackrow, T. Fleming, H. Trewhitt, T. Ellis, and G.S. Baldwin. 2015. BASIC: A New Biopart Assembly Standard for Idempotent Cloning Provides Accurate, Single-Tier DNA Assembly for Synthetic Biology. ACS Synth. Biol. 4:781-787.

14. Sarrion-Perdigones, A., M. Vazquez-Vilar, J. Palací, B. Castelijns, J. Forment, P. Ziarsolo, J. Blanca, A. Granell, and D. Orzaez. 2013. GoldenBraid 2.0: a comprehensive DNA assembly framework for plant synthetic biology. Plant Physiol. 162:1618-1631.

15. De Paoli, H.C., G.A. Tuskan, and X. Yang. 2016. An innovative platform for quick and flexible joining of assorted DNA fragments. Sci. Rep. 6:19278.

Received 08 December 2016; accepted 24 May 2017.

Address correspondence to Anthony Hall, The Earlham Institute, Norwich Research Park, Norfolk, NR4 7UH, UK. E-mail: anthony.hall@Earlham.ac.uk 